Original Paper http://ajol.info/index.php/ijbcs http://indexmedicus.afro.who.int

\title{
Phénologie florale et production fruitière de Syzygium guineense (Willd.) DC. subsp. macrocarpum (Myrtaceae) en zone soudano-guinéenne au Bénin
}

\author{
Romaël Badjrêhou BADOU *, Hounnankpon YEDOMONHAN, \\ Aristide Cossi ADOMOU et Akpovi AKOEGNINOU
}

\author{
Laboratoire de Botanique et Ecologie Végétale, Département de Biologie Végétale, Faculté des Sciences et \\ Techniques, Université d'Abomey-Calavi (UAC), 01 BP 4521 Cotonou, Bénin. \\ *Auteur correspondant ; E-mail : romaelbadou@gmail.com /romaelbadou@yahoo.com
}

\section{REMERCIEMENTS}

Les auteurs remercient très sincèrement la Fondation Internationale pour la Science (FIS) pour avoir financé cette recherche à travers la bourse D/4014-2 octroyée au co-auteur de cet article.

\section{RESUME}

Les variations phénologiques sont quelquefois considérées comme une stratégie d'adaptation écologique et physiologique des espèces à leur environnement. La présente étude a eu pour objectif de déterminer les phénophases florales et la production en fruits de Syzygium guineense subsp. macrocarpum sur un échantillonnage de 3 forêts naturelles. Les données collectées ont porté sur l'identification et la caractérisation des stades de floraison et de fructification, la quantification de la production fruitière et les caractéristiques dendrométriques des individus. Au total, $85 \%$ des arbres et arbustes ont fleuri pendant la saison sèche malgré le stress des feux. Ces individus en état de reproduction ont des diamètres compris entre 2,07 et 42,04 cm et des hauteurs allant de 1,50 à 11,50 m. Quatre stades de floraison (fleurs initiées, boutons floraux, fleurs épanouies, fleurs sénescentes) et quatre stades de fructification (fruits initiés, jeunes fruits, fruits développés, fruits mûrs) sont nettement distingués durant 116 à 128 jours. En sommes, la floraison de $S$. guineense subsp. macrocarpum est abondante (110330 fleurs initiées/ arbre) mais sa production fruitière est relativement faible (89 à 654 fruits mûrs/arbre).

(C) 2017 International Formulae Group. All rights reserved.

Mots clés : Bénin, Syzygium guineense, phénophases, production fruitière.

\begin{abstract}
The phenological variations are sometimes considered as a strategy of ecological and physiological adaptation of the species to their environment. The purpose of this study was to determine the floral phenophases and fruit production of $S$. guineense subsp. macrocarpum on a sampling of 3 natural forests. The data collected focused on the identification and characterization of the flowering and fruiting stages, the quantification of fruit production and the dendrometric characteristics of individuals. A total of $85 \%$ of trees and shrubs having bloomed during the dry season despite fire stress. These individuals in the state of reproduction have diameters between 2.07 and $42.04 \mathrm{~cm}$ and heights ranging from 1.50 to $11.50 \mathrm{~m}$. Four stages of flowering (floral initiation, developed bud, open flower and senescent flowers) and four stages of fruiting (fruits set, young fruits in development, fruits well developed and mature fruits) are clearly distinguished
\end{abstract}


during 116 - 128 days. In conclusion, the flowering of $S$. guineense subsp. macrocarpum is abundant (110330 initiated flowers/ tree) but its fruit production is relatively low (89 à 654 mature fruits /tree).

(C) 2017 International Formulae Group. All rights reserved.

Keywords: Benin, Syzygium guineense, phenophases, fruit production.

\section{INTRODUCTION}

Beaucoup d'espèces d'arbres intéressantes pour l'alimentation humaine sont rencontrées sur les marchés locaux et régionaux africains sans que leur potentiel de production en fruits ne soit connu (Boffa, 2000). Cela crée d'énormes contraintes pour leur valorisation. En effet, les cycles phénologiques des arbres tropicaux sont influencés par des facteurs biotiques, climatiques et édaphiques et la disponibilité de données détaillées sur les modes de floraison et de fructification est importante pour permettre une gestion rationnelle des ressources génétiques forestières (Chuine et al., 2000). Les variations phénologiques observées dans le temps sont quelquefois considérées comme une stratégie d'adaptation écologique et physiologique des espèces à leur environnement, et sont susceptibles de promouvoir l'allopollinisation (Boshier, 2000).

$\mathrm{Au}$ Bénin, l'apiculture est toujours considérée comme une activité secondaire à cause de sa faible rentabilité économique malgré l'appropriation par les agroapiculteurs des techniques modernes de production (Yédomonhan et al., 2009). Les travaux de Yédomonhan (2009) ont montré que la promotion de l'apiculture est subordonnée à la disponibilité, dans l'environnement des ruches, des espèces végétales mellifères dont les fleurs produisent une forte quantité de nectar. Au nombre de ces espèces à forte production nectarifère, figure Syzygium guineense (Willd.) DC. qui est l'espèce dont la production de nectar est la plus élevée en zone soudano-guinéenne au Bénin (7,70-7,75 $\mu \mathrm{l} /$ fleur/heure au cours des heures de forte sécrétion nectarifère) (Yédomonhan, 2009).

Des peuplements de $S$. guineense offriraient alors un rendement en miel élevé des ruches car elle est scientifiquement reconnue comme espèce à forte source de nectar pour les abeilles (Djonwangwe et al.,
2011). Malheureusement, sa densité dans les écosystèmes est très faible, avec une valeur comprise entre 0,96 tige/ha en forêt claire et 5,68 tiges/ha en forêt dense sèche en zone soudano-guinéenne au Bénin (Badou et Yédomonhan, 2017). Il apparaît donc claire que $S$. guineense a en forêt claire une densité en dessous de celle de Afzelia africana $(1,27$ tige/ha) considérée comme espèce menacée de disparition au Bénin (Adomou, 2005) et vulnérable sur la liste rouge de l'UICN (2000). Pourtant, la production florale de l'espèce a été évaluée à 45 fleurs/inflorescence et 57746 fleurs/arbre par Yédomonhan (2009) contre une régénération moyenne de 2,72 tiges/ha et une densité de 2,69 tiges/ha pour les individus de dbh d'au moins $10 \mathrm{~cm}$ et 2,43 tiges/ha pour les individus de moins de $10 \mathrm{~cm}$ de diamètre (Badou et Yédomonhan, 2017). La densité d'individus de $S$. guineense reste nettement en dessous de la production florale de l'espèce. Il est donc nécessaire de comprendre la biologie et l'écologie de la reproduction de l'espèce. L'objectif de ce travail était donc de déterminer les phénophases florales et évaluer la production fruitière de Syzygium guineense afin d'envisager son éventuelle domestication à des fins apicoles.

\section{MATERIEL ET METHODES Milieu d'étude}

Le milieu d'étude est la zone de transition soudano-guinéenne qui est subdivisée en trois secteurs phytogéographiques dont le secteur central sud à climax de type forêt dense sèche, le secteur central nord à climax de type miombo et le secteur central nord-ouest qui comporte des îlots de forêt dense humide semi-décidue sèche (Akoègninou et al., 2006). Le climat est de type tropical humide de transition avec une pluviosité annuelle de 1200 à $1300 \mathrm{~mm}$ d'eau. Les sols sont du type ferrugineux et ferralitique. La végétation est une mosaïque 
de forêt claire, de forêt dense sèche, de forêt dense humide semi-décidue, de savanes arborée et arbustive et de galerie forestière. L'agriculture est la principale activité réalisée dans le milieu d'étude.

Trois (03) forêts classées ont été retenues dans la zone. Ces dernières ont été choisies de façon à avoir une forêt classée à l'ouest, une autre à l'est et une dernière au nord de la zone d'étude; le sud étant dépourvu de forêt classée. Il s'agit des forêts classées de Bassila à l'Ouest, de Tchaourou à l'Est et de N'Dali au Nord de la zone d'étude (Figure 1).

\section{Méthode}

Les données ont été collectées durant 2 années consécutives. Les périodes de collecte des données sont du 17 décembre 2015 au 02 mai 2016 et du 15 décembre 2016 au 30 avril 2017 dans les 3 forêts retenues. A cet effet, 10 arbres reproductifs (espacés d'au moins 13 $\mathrm{m})$ et 10 inflorescences/arbre reproductif ont été choisis au hasard et marqués. Les observations phénologiques ont eu lieu tous les 2 jours dès l'initiation florale jusqu'au stade de fruits mûrs, afin d'identifier les stades phénologiques et de collecter les données quantitatives liées aux divers organes (fleurs et fruits).

Les différents stades de floraison et de fructification de $S$. guineense ont été identifiés sur la base des caractères morphologiques qualitatifs (couleur, caducité ou non, mise en place) des pièces. Ces caractères qualitatifs ont été complétés par les caractères quantitatifs pour mieux caractériser les différents stades de reproduction. Pour ce faire, 5 fleurs/inflorescence et 5 fruits/infrutescence ont été prélevés à chaque stade identifié sur 3 inflorescences ou 3 infrutescences sur les 10 arbres marqués au préalable, soit donc 15 fleurs et 15 fruits échantillonnés par stade de reproduction. Les données collectées ont portées sur la longueur et le diamètre des pièces des organes. Le diamètre des fleurs a été pris au niveau du réceptacle floral. Toutes ces observations, notations et mesures ont été faites de $8 \mathrm{~h}$ à $15 \mathrm{~h}$.

Les inventaires dendrométriques des arbres en production ont eu lieu dans 25 placeaux contigus de $100 \mathrm{~m}$ x $100 \mathrm{~m}$ par site, soit 75 placeaux d'un hectare (ha) pour les 3 sites. A l'intérieur de chaque placeau, les paramètres collectés par arbre ou arbuste étaient : le diamètre à $1,30 \mathrm{~m}$ du sol (dbh), la hauteur totale, l'état phénologique et les coordonnées géographiques.

\section{Analyses statistiques}

Les différents stades de phénophases reproductrices ont établis et leurs caractéristiques sont été présentées sur la base des caractères morphologiques qualitatifs (couleur, présence ou non) et quantitatifs (longueur et diamètre) ont été présentées par inflorescence ou infrutescence et par arbre. L'analyse de variance (ANOVA) a permis de vérifier la variabilité de ces caractères quantitatifs d'un arbre à l'autre et d'un site à l'autre à l'aide du logiciel MINITAB 14. Les valeurs moyennes obtenues par stade de phénophases ont été comparées 2 à 2 à l'aide du test de student (t-test).

Le synchronisme $\mathrm{Z}$ de chacun des 3 populations a été déterminé suivant la formule utilisée par Ewédjè et al. (2015). Il varie de 0 à 1 et mesure de la superposition de la durée totale de la floraison de tous les individus avec la durée de la floraison de chaque individu de la population. Plus $\mathrm{Z}$ est proche de 1, plus les individus de la population considérée fleurissent presque au même moment. Elle a été évaluée avec le stade de fleurs épanouies. Cet indice est donné par la formule suivante :

$$
Z=(1 / n) \sum_{i=1}^{n}\left(\left[\frac{1}{n-1}\right]\left[\frac{1}{f i}\right] \sum_{j \neq i}^{n} E_{i j}\right), \text { avec : }
$$

$\mathrm{n}=$ nombre d'individus de la population, $\mathrm{fi}=$ nombre de jours de floraison de l'individu i, Eij $=$ nombre de jours durant lesquels $\mathrm{i}$ et $\mathrm{j}$ fleurissent de façon synchronisée.

La production de fruits par ha $\left(\mathrm{P}_{\mathrm{T}}\right)$ a été estimée en utilisant la production moyenne d'un arbre et la densité de plantation $\left(D_{p}\right)$ de l'espèce (Kouyaté et al., 2006).

$$
\mathrm{P}_{\mathrm{T}}=\left(\sum \mathrm{pi} / \mathrm{N}\right) \times\left(\sum \mathrm{ni} / 10000\right) \text {, où pi est }
$$

la quantité de fruits quantifiés sur l'arbre i et $\mathrm{N}$ est le nombre total d'arbres sur lesquels les fruits ont été quantifiés, ni est le nombre de tiges par placeau et $10000 \mathrm{~m}^{2}=1$ ha. 


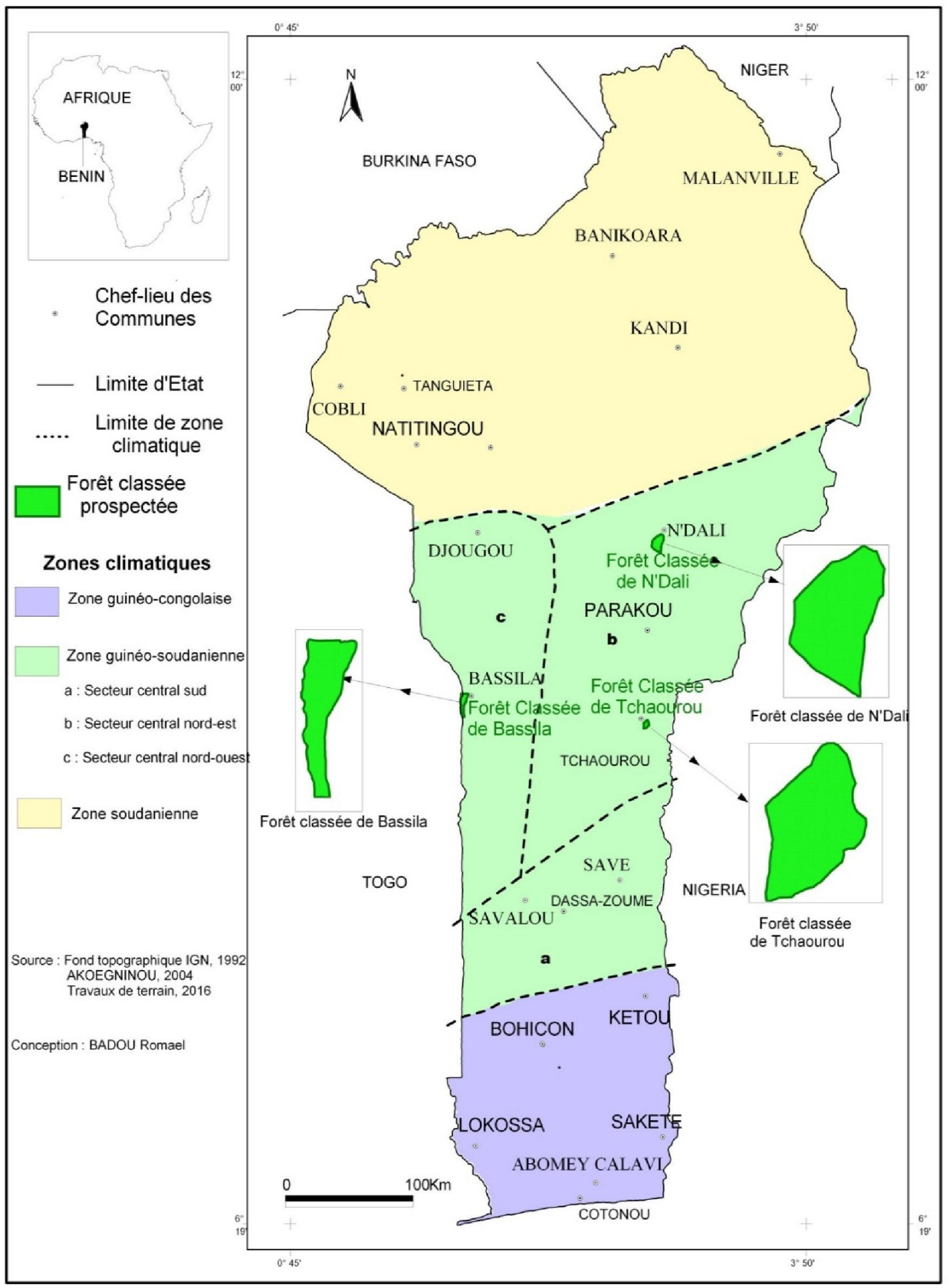

Figure 1 : Localisation géographique des 3 forêts classées explorées en zone soudano-guinéenne au Bénin. 


\section{RESULTATS}

\section{Caractéristiques des stades de floraison et de fructification chez $S$. guineense subsp. macrocarpum}

$\mathrm{Au}$ total, 8 stades de phénophases reproductrices ont été enregistrés dont 4 au cours de la floraison et 4 pour la fructification (Tableau 1). Au cours de la floraison, les fleurs initiées $\left(\mathrm{fl}_{1}\right)$ s'individualisent véritablement en fleurs véritables avec la différenciation des pièces périanthaires au cours du deuxième stade $\left(\mathrm{fl}_{2}\right)$. Le troisième stade $\left(\mathrm{fl}_{3}\right)$ se caractérise par le pic de développement floral avec l'ouverture complète et l'acquisition de la longueur maximale $(15,10 \pm 0,88 \mathrm{~mm})$ de la fleur. Il marque aussi la fin de la croissance et du développement des étamines. Enfin, le dernier stade floral est celui de la sénescence $\left(\mathrm{fl}_{4}\right)$ qui traduit la chute des étamines. Les dimensions des pièces florales sont similaires, d'un arbre à l'autre et d'un site à l'autre à un même stade $(p>0,05)$. Par contre, elles varient très significativement d'un stade à l'autre.

En ce qui concerne les stades de fructification, la nouaison $\left(\mathrm{fr}_{1}\right)$ se matérialise par un léger gonflement de l'ovaire fécondé et dépourvu totalement des autres pièces florales. Ce premier stade est suivi de celui des jeunes fruits $\left(\mathrm{fr}_{2}\right)$ chez qui la forme du fruit s'individualise. C'est au stade de fruit développé ( $\left.\mathrm{fr}_{3}\right)$ que l'accroissement diamétrique $\mathrm{du}$ fruit est très remarquable $(19,00 \pm 3,65 \mathrm{~mm})$. Enfin, le dernier stade est celui du fruit mûr $\left(\mathrm{fr}_{4}\right)$ caractérisé par un péricarpe à couleur passant du vert-clair au vert-jaunâtre ou au violet.

L'analyse de variance a montré que les dimensions (longueur et diamètre) des fruits ne varient significativement ni d'un arbre à l'autre, ni d'un site à l'autre $(\mathrm{p}>0,05)$ pour chacun des stades de fructification. Par contre, les moyennes de la longueur et du diamètre diffère significativement d'un stade de phénophase reproductrice au stade consécutif.

\section{Calendrier des phénophases reproductrices} et digramme de reproduction de $S$. guineense subsp. macrocarpum dans trois forêts

Considérant les 2 années d'observation, la floraison de $S$. guineense s'est étalée entre la période de 20 décembre 2015 et 10 mars 2016, soit une durée totale de 81 jours. Dans la $2^{\text {ième }}$ année, elle s'est étalée entre la période de 18 décembre 2016 et 19 février 2017, soit une durée totale de 65 jours. Toutefois, elle s'est déroulée de façon différée au sein des trois sites; sa durée étant de 75 jours en 2016 et 64 jours en 2017 à Bassila. A N'Dali, elle a duré 60 jours en 2016 et 61 jours en 2017 ; et à Tchaourou 65 jours en 2016 et 63 jours en 2017 (Tableau 2). En considérant les différents stades de phénophase florale, leur durée est partout identique (10 jours) pour les stades de boutons floraux et 10 à 15 jours pour le stade de fleurs épanouies. Par contre, le stade de fleurs initiées présente une durée élevée, 25 à 30 jours. Aussi, le stade de fleurs sénescentes qui n'a duré que 14 à 15 jours en 2017, a été très long (30 jours) à Bassila en 2016.

S'agissant de la fructification, elle s'est déroulée du 20 février au 30 avril 2016 dans la $1^{\text {ère }}$ année, soit une durée de 70 jours. Dans la $2^{\text {ième }}$ année, elle a démarré le 17 février pour atteindre la phase des fruits mûrs le 25 avril, soit une durée 67 jours (Tableau 2). Mais, il existe un léger décalage entre le développement des divers stades de fructification entre les 3 différents peuplements. Ainsi, la durée de la fructification est au totale de 55 à 60 jours à N'Dali, 53 à 55 jours à Tchaourou et 50 à 64 jours à Bassila. Au total, la formation des fruits mûrs a nécessité, depuis l'initiation 
florale, 116 à 120 jours (4 mois) à N'Dali et Tchaourou puis 125 à 128 jours à Bassila. Les peuplements de Tchaourou et de N'Dali donnent précocement les fruits matures, respectivement 18 et 20 avril en 2016 , et à même date ( 13 avril) en 2017 ; alors que celui de Bassila donne tardivement les fruits mûrs à la date du 30 avril en 2016 et 25 avril en 2017 (Tableau 2).

Le test de chi-2 a révélé l'existence d'une forte indépendance entre le nombre de jours pour chaque phase phénologique. Il n'est pas statistiquement influencé par l'année (ddl $\left.=20 ; \mathrm{x}^{2}=11,919 ; \mathrm{p}=0,919\right)$, ni par le site $\left(\mathrm{ddl}=26 ; \mathrm{x}^{2}=14,312 ; \mathrm{p}=0,969\right)$.

L'indice de synchronisme floral (Z) de l'espèce est de 0,94 dans la population de Bassila et 0,93 dans les populations de N'Dali et de Tchaourou. Ces indices montrent que la floraison se déroule simultanément au sein de chaque peuplement. Cela indique que dans chaque station, on a une bonne superposition des périodes de floraison des différents arbres les uns par rapport aux autres.

La Figure 2 présente les proportions d'individus de $S$. guineense subsp. macrocarpum en reproduction dans ses phytocénoses à Bassila, N'Dali et Tchaourou en 2015-2016 et 2016-2017. La superposition de ces courbes indique que tous les arbres et arbustes de $S$. guineense subsp. macrocarpum $(100 \%)$ ont produit pleinement du 18 décembre au 30 avril à Bassila contre $71 \%$ et $72 \%$ respectivement à N'Dali et Tchaourou aussi bien en 2015-2016 qu'en 2016-2017. Quel que soit le site ou l'année, la pleine floraison de l'espèce se situe en janvierfévrier et la pleine fructification en mars-avril.

\section{Densité des organes reproducteurs par stade de phénophases reproductrices \\ Le nombre moyen d'organes reproducteurs comptés par}

inflorescence/infrutescence et par arbre à chacun des 8 stades de phénophases reproductrices est récapitulé dans le Tableau 3. Le nombre de fleurs initiés/inflorescence est compris entre 444 à Bassila et 501 à Tchaourou, avec une moyenne 477 pour la zone d'étude. Parmi les 477 fleurs initiées en moyenne par inflorescence, seulement 2 arrivent au stade de fruit mûr par infrutescence. Cette tendance globale est aussi observée au niveau de chacun des trois sites. Le nombre moyen d'organes reproducteurs dénombrés par inflorescence diffère significativement d'une localité à l'autre pour 3 stades de phénophases, à savoir : le stade de fleurs initiées, le stade de fruits développés et le stade de fruits mûrs. Par contre, il est similaire au niveau de N'Dali et Tchaourou pour les stades restants à l'exception du stade de boutons floraux où c'est plutôt Bassila et Tchaourou qui enregistrent des valeurs presque identiques (68 boutons/inflorescence à Bassila et 69 boutons floraux/inflorescence à Tchaourou).

A l'échelle des arbres, le nombre moyen de fleurs initiées par arbre varie de 56460 à Tchaourou à 193279 à Bassila, avec une moyenne de 110330 pour la zone d'étude (Tableau 3). De ces dernières, seulement 290 fleurs atteignent le stade de fruits mûrs. Cette baisse considérable d'organes reproducteurs entre les 2 extrêmes phases de phénologie reproductrice est aussi enregistrée dans chacun des 3 sites d'études. De plus, le nombre moyen d'organes reproducteurs dénombrés par arbre diffère significativement d'une localité à l'autre pour tous les huit stades de phénophases identifiés. L'importante fructification enregistrée (654 fruits/ arbre) à Bassila contre 89 et 124 fruits/arbre respectivement à N'Dali et Tchaourou est due à la bonne floraison des arbres dans la forêt classée de Bassila 
comportant beaucoup d'individus à gros diamètres. A cet effet, un seul arbre échantillonné a produit $413 \pm 196$ inflorescences aboutissant à $314 \pm 131$

infrutescences à Bassila; $147 \pm 102$ inflorescences aboutissant à $91 \pm 80$ infrutescences à N'Dali et $101 \pm 47$ inflorescences aboutissant à $48 \pm 38$ infrutescences/arbre dans la forêt classée de Tchaourou.

Evolution de la perte en organes fructifères et taux de fructification de Syzygium guineense subsp. macrocarpum au Bénin

La Figure 3 indique la perte moyenne cumulée d'organes reproducteurs par arbre depuis le stade de fleur initiée jusqu'au stade de fruits mûrs. Elle a une allure logarithmique, avec une très forte ascendance entre le stade de fleurs initiées et celui de bouton floral développé où le taux d'avortement est de 84,19\%. Par la suite, ce taux d'avortement croît faiblement jusqu'au stade de fruit mûr. Ainsi, le taux cumulé moyen d'avortement d'organes reproducteurs entre les stades $\mathrm{fl}_{1}$ (fleurs initiées) et $\mathrm{fr}_{4}$ (fruits mûrs) est de 99,74\%. Par conséquent, seulement 0,26\% de fleurs initiées par pied de $S$. guineense subsp. macrocarpum arrivent à se développer en fruits mûrs au terme du développement des organes reproducteurs.

Caractéristiques structurales de la population de $S$. guineense subsp. macrocarpum et production de fruits

Au total, 85\% des arbres et arbustes de S. guineense subsp. macrocarpum ont fleuri dans la zone d'étude. Le pourcentage d'individus ayant fleuri/site est de $100 \%$ à Bassila, $71 \%$ à $N^{\prime}$ Dali et de $72 \%$ à
Tchaourou. Ces individus en état de reproduction ont des diamètres compris entre 2,07 et 42,04 cm et des hauteurs allant de 1,50 à $11,50 \mathrm{~m}$ en considérant 1'ensemble des 3 sites. Toutefois, les valeurs minimales et maximales de diamètres et de hauteurs enregistrées pour les arbres et arbustes fertiles varient d'un site à l'autre. De plus, elles ne déterminent en rien l'état phénologique des plants. Par exemple, parmi les 15\% d'individus en état végétatif, seulement 5 (soit $1,30 \%$ ) ont des valeurs de diamètre et de hauteur inférieures à celles obtenues pour les plants en fleurs. Les $13,70 \%$ restants ont les variables structurales comprises entre les intervalles obtenus pour les arbres reproductifs mais sont pourtant en étant végétatif.

La production fruitière par individu de S. guineense subsp. macrocarpum a varié en moyenne de 89 à 654 fruits mûrs /arbre selon l'individu selon le site, avec une moyenne de 291 fruits/ha pour l'ensemble des 3 sites. Ceci correspond à une production moyenne comprise entre 281 à 4657 fruits mûrs/ha, respectivement entre N'Dali et Bassila. Les peuplements de la forêt classée de Bassila ont donné une meilleure production qui a été statistiquement supérieure $(p<0,05)$ à celui des arbres des forêts classées de N'Dali et Tchaourou (Tableau 4).

Le nombre de fruits par arbre est significativement influencé par le diamètre des individus $(\mathrm{ddl}=59 ; \mathrm{p}=0,000)$ et par la hauteur $(\mathrm{ddl}=59 ; \mathrm{p}=0,000)$. De plus, le poids des fruits peut être également déterminé par le diamètre des arbres et arbustes $(\mathrm{ddl}=$ $59 ; \mathrm{p}=0,000)$ et par leur hauteur $(\mathrm{ddl}=59 ; \mathrm{p}$ $=0,000)$. 


\section{R. B. BADOU et al. / Int. J. Biol. Chem. Sci. 11(5): 2466-2480, 2017}

Tableau 1 : Caractéristiques morphologiques des stades de phénophases reproductrices de $S$. guineense subsp. macrocarpum.

\begin{tabular}{|c|c|c|c|c|}
\hline \multirow{2}{*}{$\begin{array}{l}\text { Phénophases } \\
\text { reproductrices }\end{array}$} & \multirow[b]{2}{*}{ Stades } & \multirow[b]{2}{*}{ Caractères qualitatifs } & \multicolumn{2}{|c|}{ Caractères quantitatifs } \\
\hline & & & $\begin{array}{l}\text { longueur de la } \\
\text { fleur }(\mathrm{mm})\end{array}$ & $\begin{array}{c}\text { diamètre de la } \\
\text { fleur }(\mathrm{mm})\end{array}$ \\
\hline \multirow{4}{*}{ Floraison } & Fleurs initiées $\left(\mathrm{fl}_{1}\right)$ & Fleurs minuscules, périanthe non distinct. & $5,42 \pm 0,61^{\mathrm{a}}$ & $2,61 \pm 0,45^{\mathrm{a}}$ \\
\hline & Boutons floraux $\left(\mathrm{fl}_{2}\right)$ & $\begin{array}{l}\text { Fleurs coniques, non ouvertes, calice vert, corolle rouge ou vert-clair et visible } \\
\text { seulement par le sommet de la fleur. }\end{array}$ & $7,93 \pm 0,82^{b}$ & $4,29 \pm 0,51^{b}$ \\
\hline & Fleurs épanouies $\left(\mathrm{fl}_{3}\right)$ & $\begin{array}{l}\text { Fleurs entièrement ouvertes, calice persistant, corolle absente (détachée), } \\
\text { androcée complet, filet blanc, anthère jaune, style et stigmate blancs et presque } \\
\text { cachés par les étamines. }\end{array}$ & $15,10 \pm 0,88^{\mathrm{c}}$ & $6,05 \pm 0,51^{\mathrm{c}}$ \\
\hline & Fleurs sénescentes $\left(\mathrm{fl}_{4}\right)$ & $\begin{array}{l}\text { Fleurs entièrement ouvertes, calice persistant, corolle absente (détachée), } \\
\text { androcée fané et à étamines partiellement détachées, gynécée entièrement nu } \\
\text { et à ovaire infère. }\end{array}$ & $9,62 \pm 1,25^{\mathrm{d}}$ & $5,88 \pm 0,92^{\mathrm{d}}$ \\
\hline \multirow[t]{4}{*}{ Fructification } & Fruits initiés $\left(\mathrm{fr}_{1}\right)$ & $\begin{array}{l}\text { Ovaire légèrement gonflé, absence totale des pièces florales, calice transformé } \\
\text { en une coupe au sommet de l'ovaire fécondé. }\end{array}$ & $12,33 \pm 2,19^{\mathrm{a}}$ & $6,33 \pm 0,83^{\mathrm{a}}$ \\
\hline & Jeunes fruits $\left(\mathrm{fr}_{2}\right)$ & $\begin{array}{l}\text { Gonflement un peu plus prononcé de l'ovaire fécondé, individualisation de la } \\
\text { forme du fruit. }\end{array}$ & $16,48 \pm 2,31^{b}$ & $9,40 \pm 1,79^{b}$ \\
\hline & Fruits développés $\left(\mathrm{fr}_{3}\right)$ & Fruits à gonflement bien prononcé, vert-clairs. & $22,27 \pm 2,87^{\mathrm{C}}$ & $19,00 \pm 3,65^{\mathrm{c}}$ \\
\hline & Fruits mûrs $\left(\mathrm{fr}_{4}\right)$ & Couleur du péricarpe devenant vert-jaunâtre ou violette & $24,73 \pm 2,53^{\mathrm{d}}$ & $21,36 \pm 3,28^{\mathrm{d}}$ \\
\hline
\end{tabular}


Tableau 2 : Variation du calendrier de floraison et de fructification au sein des sites en 2016 et 2017.

\begin{tabular}{|c|c|c|c|c|c|c|}
\hline \multirow[b]{2}{*}{ Stades phénologiques } & \multicolumn{2}{|c|}{ Bassila } & \multicolumn{2}{|c|}{ N'Dali } & \multicolumn{2}{|c|}{ Tchaourou } \\
\hline & $\begin{array}{c}\text { Date de } \\
\text { démarrage }\end{array}$ & $\begin{array}{c}\text { Durée } \\
\text { (jours) }\end{array}$ & $\begin{array}{c}\text { Date de } \\
\text { démarrage }\end{array}$ & $\begin{array}{c}\text { Durée } \\
\text { (jours) }\end{array}$ & $\begin{array}{c}\text { Date de } \\
\text { démarrage }\end{array}$ & $\begin{array}{c}\text { Durée } \\
\text { (jours) }\end{array}$ \\
\hline \multicolumn{7}{|c|}{ Année 2016} \\
\hline Fleurs initiées $\left(\mathrm{fl}_{1}\right)$ & $27 / 12 / 15$ & 25 & $22 / 12 / 15$ & 25 & $20 / 12 / 15$ & 30 \\
\hline Boutons floraux $\left(\mathrm{fl}_{2}\right)$ & $21 / 01 / 16$ & 10 & $16 / 01 / 16$ & 10 & $19 / 01 / 16$ & 10 \\
\hline Fleurs épanouies $\left(\mathrm{fl}_{3}\right)$ & $31 / 01 / 16$ & 10 & $26 / 01 / 16$ & 10 & $29 / 01 / 16$ & 10 \\
\hline Fleurs sénescentes $\left(\mathrm{fl}_{4}\right)$ & $10 / 02 / 16$ & 30 & $05 / 02 / 16$ & 15 & $08 / 02 / 16$ & 15 \\
\hline Fruits initiés $\left(\mathrm{fr}_{1}\right)$ & $11 / 03 / 16$ & 15 & $20 / 02 / 16$ & 15 & $23 / 02 / 16$ & 15 \\
\hline Jeunes fruits $\left(\mathrm{fr}_{2}\right)$ & $26 / 03 / 16$ & 30 & $06 / 03 / 16$ & 35 & $09 / 03 / 16$ & 30 \\
\hline Fruits développés $\left(\mathrm{fr}_{3}\right)$ & $25 / 04 / 16$ & 5 & $10 / 04 / 16$ & 10 & $08 / 04 / 16$ & 10 \\
\hline Fruits mûrs $\left(\mathrm{fr}_{4}\right)$ & $30 / 04 / 16$ & - & $20 / 04 / 16$ & - & $18 / 04 / 16$ & - \\
\hline Total & \multicolumn{2}{|c|}{125} & \multicolumn{2}{|c|}{120} & \multicolumn{2}{|c|}{120} \\
\hline \multicolumn{7}{|c|}{ Année 2017} \\
\hline Fleurs initiées $\left(\mathrm{fl}_{1}\right)$ & $18 / 12 / 16$ & 25 & $18 / 12 / 16$ & 25 & $18 / 12 / 16$ & 25 \\
\hline Boutons floraux $\left(\mathrm{fl}_{2}\right)$ & $12 / 01 / 17$ & 10 & $12 / 01 / 17$ & 10 & $12 / 01 / 17$ & 10 \\
\hline Fleurs épanouies $\left(\mathrm{fl}_{3}\right)$ & $22 / 01 / 17$ & 15 & $22 / 01 / 17$ & 11 & $22 / 01 / 17$ & 14 \\
\hline Fleurs sénescentes $\left(\mathrm{fl}_{4}\right)$ & $06 / 02 / 17$ & 14 & $02 / 02 / 17$ & 15 & $05 / 02 / 17$ & 14 \\
\hline Fruits initiés $\left(\mathrm{fr}_{1}\right)$ & $20 / 02 / 17$ & 20 & $17 / 02 / 17$ & 13 & $19 / 02 / 17$ & 11 \\
\hline Jeunes fruits $\left(\mathrm{fr}_{2}\right)$ & $12 / 03 / 17$ & 34 & $02 / 03 / 17$ & 32 & $02 / 03 / 17$ & 29 \\
\hline Fruits développés $\left(\mathrm{fr}_{3}\right)$ & $15 / 04 / 17$ & 10 & $03 / 04 / 17$ & 10 & $31 / 03 / 17$ & 13 \\
\hline Fruits mûrs $\left(\mathrm{fr}_{4}\right)$ & $25 / 04 / 17$ & - & $13 / 04 / 17$ & - & $13 / 04 / 17$ & - \\
\hline Total & \multicolumn{2}{|c|}{128} & \multicolumn{2}{|c|}{116} & \multicolumn{2}{|c|}{116} \\
\hline
\end{tabular}

Tableau 3: Variation du nombre d'organes reproducteurs par stade de phénophases reproductrices.

\begin{tabular}{|c|c|c|c|c|}
\hline \multirow{2}{*}{$\begin{array}{l}\text { Stades de phénophases } \\
\text { reproductrices }\end{array}$} & \multicolumn{4}{|c|}{ Sites d'étude } \\
\hline & Bassila & N'Dali & Tchaourou & Moyenne \\
\hline \multicolumn{5}{|c|}{ A l'échelle de l'inflorescence ou de l'infrutescence } \\
\hline Fleurs initiées $\left(\mathrm{fl}_{1}\right)$ & $468 \pm 132^{\mathrm{a}}$ & $444 \pm 117^{\mathrm{b}}$ & $501 \pm 146^{\mathrm{c}}$ & $477 \pm 121$ \\
\hline Boutons floraux $\left(\mathrm{fl}_{2}\right)$ & $68 \pm 17^{\mathrm{a}}$ & $53 \pm 4^{\mathrm{b}}$ & $69 \pm 5^{\mathrm{a}}$ & $64 \pm 14$ \\
\hline Fleurs épanouies $\left(\mathrm{fl}_{3}\right)$ & $45 \pm 16^{\mathrm{a}}$ & $37 \pm 4^{b}$ & $38 \pm 9^{\mathrm{b}}$ & $41 \pm 12$ \\
\hline Fleurs sénescentes $\left(\mathrm{fl}_{4}\right)$ & $40 \pm 15^{\mathrm{a}}$ & $28 \pm 4^{b}$ & $30 \pm 8^{b}$ & $34 \pm 12$ \\
\hline Fruits initiés $\left(\mathrm{fr}_{1}\right)$ & $32 \pm 10^{\mathrm{a}}$ & $19 \pm 3^{b}$ & $22 \pm 5^{b}$ & $26 \pm 9$ \\
\hline Jeunes fruits $\left(\mathrm{fr}_{2}\right)$ & $27 \pm 8^{\mathrm{a}}$ & $10 \pm 4^{b}$ & $11 \pm 5^{b}$ & $18 \pm 10$ \\
\hline Fruits développés $\left(\mathrm{fr}_{3}\right)$ & $9 \pm 3^{\mathrm{a}}$ & $2 \pm 2^{b}$ & $6 \pm 3^{c}$ & $6 \pm 4$ \\
\hline Fruits mûrs $\left(\mathrm{fr}_{4}\right)$ & $2 \pm 1^{\mathrm{a}}$ & $1 \pm 1^{\mathrm{b}}$ & $3 \pm 2^{c}$ & $2 \pm 2$ \\
\hline \multicolumn{5}{|c|}{ A l'échelle de l'arbre } \\
\hline Fleurs initiées $\left(\mathrm{fl}_{1}\right)$ & $193279 \pm 69409^{a}$ & $73726 \pm 23956^{b}$ & $56460 \pm 22665^{c}$ & $110330 \pm 40946$ \\
\hline
\end{tabular}


R. B. BADOU et al. / Int. J. Biol. Chem. Sci. 11(5): 2466-2480, 2017

\begin{tabular}{ccccc}
\hline Boutons floraux $\left(\mathrm{fl}_{2}\right)$ & $29252 \pm 18095^{\mathrm{a}}$ & $7785 \pm 5311^{\mathrm{b}}$ & $7801 \pm 2789^{\mathrm{c}}$ & $17449 \pm 16413$ \\
\hline Fleurs épanouies $\left(\mathrm{fl}_{3}\right)$ & $19251 \pm 14072^{\mathrm{a}}$ & $5274 \pm 3300^{\mathrm{b}}$ & $4509 \pm 2721^{\mathrm{c}}$ & $11372 \pm 11969$ \\
\hline Fleurs sénescentes $\left(\mathrm{fl}_{4}\right)$ & $16340 \pm 12861^{\mathrm{a}}$ & $3852 \pm 2628^{\mathrm{b}}$ & $2569 \pm 2416^{\mathrm{c}}$ & $9151 \pm 10909$ \\
\hline Fruits initiés $\left(\mathrm{fr}_{1}\right)$ & $11492 \pm 6391^{\mathrm{a}}$ & $2259 \pm 1740^{\mathrm{b}}$ & $1706 \pm 1510^{\mathrm{c}}$ & $6276 \pm 6486$ \\
\hline Jeunes fruits $\left(\mathrm{fr}_{2}\right)$ & $9674 \pm 5265^{\mathrm{a}}$ & $1224 \pm 1047^{\mathrm{b}}$ & $608 \pm 606^{\mathrm{c}}$ & $4873 \pm 5646$ \\
\hline Fruits développés $\left(\mathrm{fr}_{3}\right)$ & $2367 \pm 1154^{\mathrm{a}}$ & $127 \pm 93^{\mathrm{b}}$ & $217 \pm 209^{\mathrm{c}}$ & $1158 \pm 1349$ \\
\hline Fruits mûrs $\left(\mathrm{fr}_{4}\right)$ & $654 \pm 317^{\mathrm{a}}$ & $89 \pm 65^{\mathrm{b}}$ & $124 \pm 152^{\mathrm{c}}$ & $290 \pm 284$ \\
\hline
\end{tabular}

Pour un stade de phénophase reproductive, les moyennes suivies de lettres différentes sont statistiquement différentes.

Tableau 4 : Variation des paramètres structuraux des individus fertiles et quantité de fruits produits par S. guineense subsp. macrocarpum.

\begin{tabular}{|c|c|c|c|c|}
\hline Paramètres & Bassila & N'Dali & Tchaourou & Site \\
\hline $\begin{array}{l}\text { Pourcentage d'individus ayant } \\
\text { fleuri }(\%)\end{array}$ & 100 & 71 & 72 & 85 \\
\hline $\begin{array}{l}\text { Intervalle de variation } \mathrm{du} \\
\text { diamètre des tiges }(\mathrm{cm})\end{array}$ & {$[6,37-42,04]$} & {$[2,07-21,97]$} & {$[2,87-30,25]$} & {$[2,07-42,04]$} \\
\hline $\begin{array}{l}\text { Intervalle de variation de la } \\
\text { hauteur des tiges }(\mathrm{m})\end{array}$ & {$[3,50-9,50]$} & {$[1,50-10,50]$} & {$[2,00-11,50]$} & {$[1,50-11,50]$} \\
\hline Nombre de fruits/arbre & $654^{\mathrm{a}}$ & $89^{\mathrm{b}}$ & $124^{\mathrm{c}}$ & 291 \\
\hline $\begin{array}{l}\text { Densité des plants fleuris } \\
\text { (tiges/ha) }\end{array}$ & $7,12^{\mathrm{a}}$ & $3,16^{\mathrm{b}}$ & $2,60^{\mathrm{c}}$ & 4,29 \\
\hline $\begin{array}{l}\text { Nombre de fruits produits par } \\
\text { hectare }\end{array}$ & $4657^{\mathrm{a}}$ & $281^{\mathrm{b}}$ & $322^{c}$ & 1244 \\
\hline
\end{tabular}

Pour un paramètre donné, les moyennes suivies de lettres différentes sont significativement différentes.

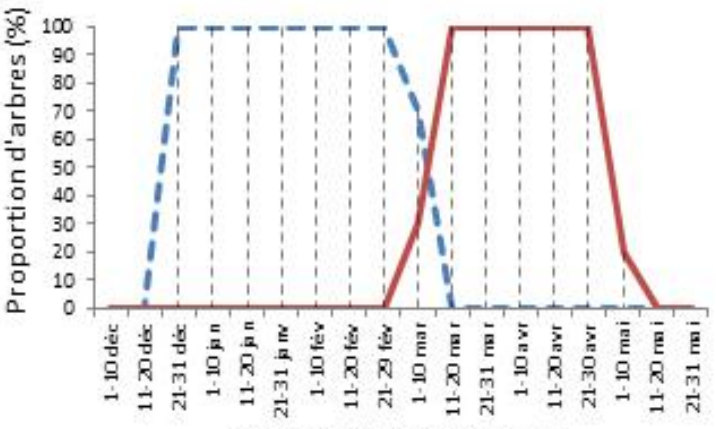

Année 2015-2016 à BASSILA

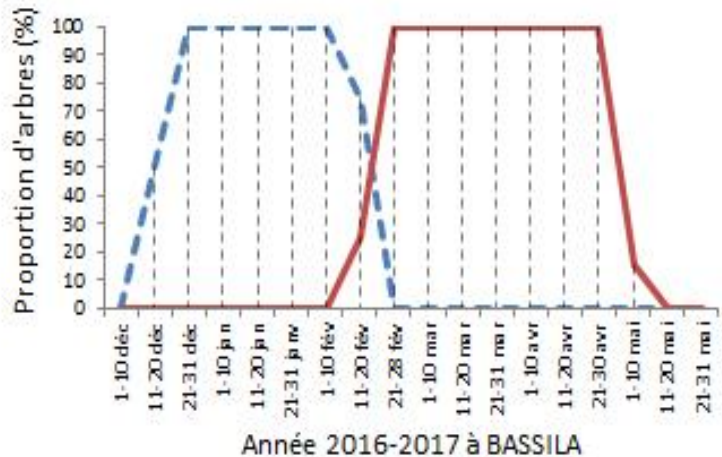

Année 2016-2017 à BASSILA 

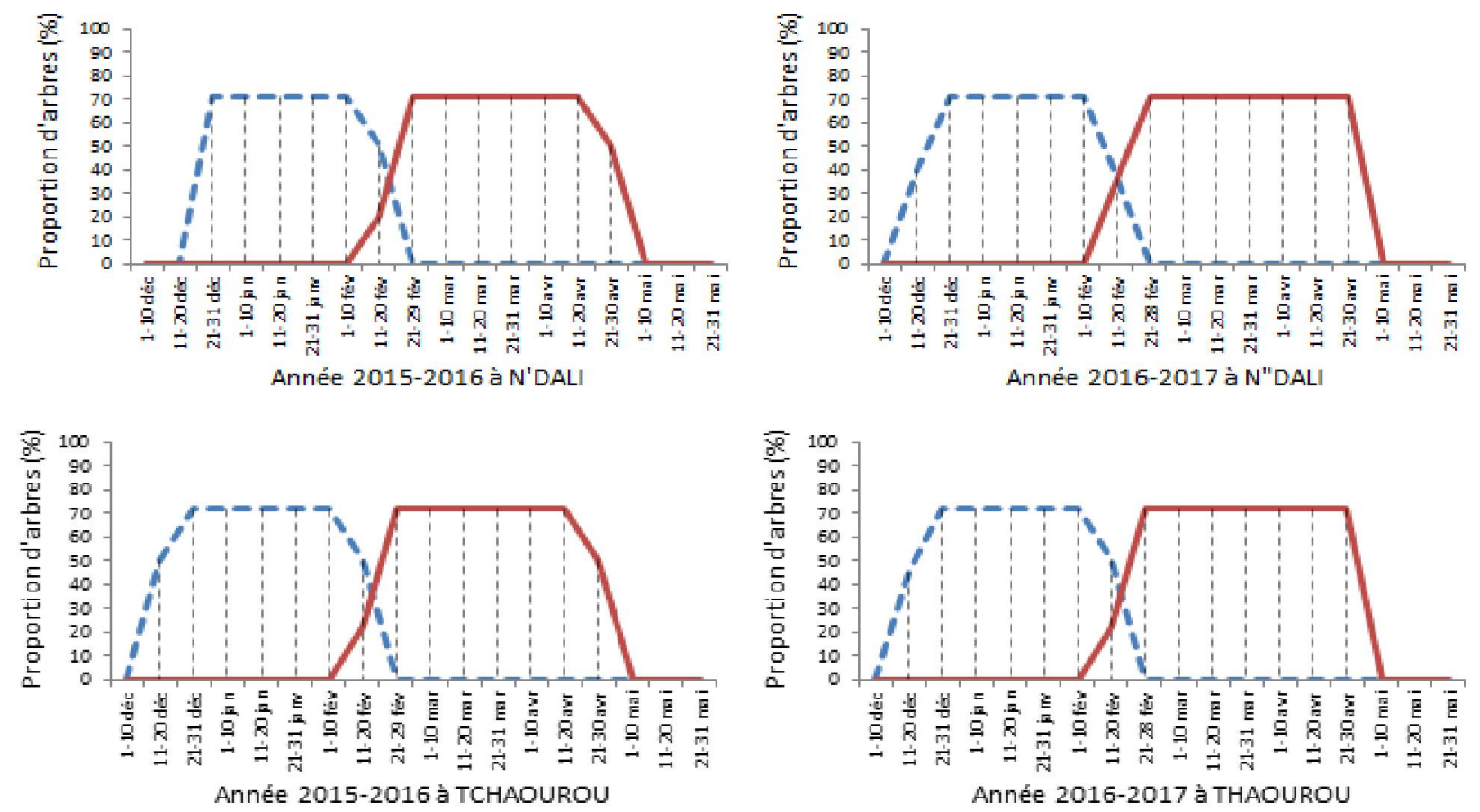

$=-=$ en floraison

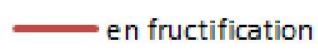

Figure 2 : Evolution temporelle des individus reproductifs de $S$. guineense subsp. macrocarpum au sein de ses peuplements.

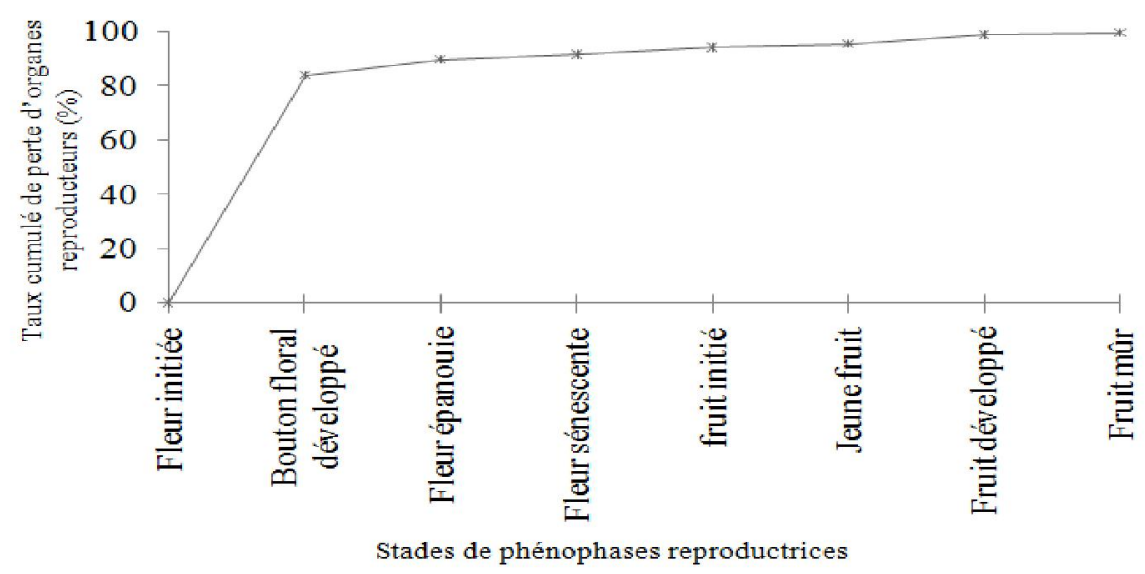

Figure 3 : Evolution du taux moyen cumulé de perte d'organes reproducteurs par arbre entre les deux extrêmes stades de phénophases reproductrices. 


\section{DISCUSSION}

Les peuplements de $S$. guineense subsp. macrocarpum étudiés sont constitués d'individus à fleurs hermaphrodites, ayant fleuri en saison sèche. La température et la pluviosité influent sur l'épanouissement total des fleurs de nombreuses espèces (Kouyaté, 2005). La précocité ou le décalage de la phénologie à divers endroits et même dans un peuplement pourrait survenir suite aux fluctuations des facteurs climatiques et édaphiques (Diouf et Zaâfouri, 2003 ; Jaouadi et al., 2012).

La floraison s'est déroulée simultanément au sein de chaque peuplement. Cette remarque générale a été confirmée par les indices de synchronisme floral qui sont relativement élevés. L'indice de synchronisme floral ( $\mathrm{Z}=0,94 ; \mathrm{N}=10)$ obtenu de $S$. guineense subsp. macrocarpum dans la forêt de Bassila est légèrement supérieur à celui obtenu pour Pentadesma butyracea $(\mathrm{Z}=0,80 ; \mathrm{N}=20)$ dans la forêt de Penessoulou (Ewédjè et al., 2015) qui se trouve dans le même secteur central nord-ouest que Bassila. En effet, Syzygium guineense fait partie de la grande diversité des plantes qui ont une pleine floraison étalée sur deux mois (janvier - février) en zone soudanoguinéenne (Yédomonhan, 2009). Cependant, aucune étude spécifique concernant Syzygium guineense subsp. macrocarpum n'a été menée. On retient seulement que dans les zones à une saison de pluie, l'espèce fleurit une fois (en saison sèche) et dans les zones à deux saisons de pluies l'espèce fleurit deux fois (Orwa et al., 2009). Le climat de la zone d'étude est du type soudano-guinéen, caractérisé par une saison sèche qui couvre la période de mi-octobre à mi-avril et une saison pluvieuse entre mi-avril et mi-octobre. Les précipitations se situent entre $1200 \mathrm{~mm}$ et $1300 \mathrm{~mm}$ avec le mois d'août comme le mois le plus pluvieux (Sinsin et Kampmann, 2010 ; Gbesso, 2014). La présente étude montrant que $S$. guineense subsp. macrocarpum a une floraison étalée entre 18 décembre et 10 mars confirme les résultats de Yédomonhan (2009), et Arbonnier (2008) qui trouvent que l'espèce fleurit en saison sèche.

Considérant les 30 arbres représentatifs, le diamètre des fruits à maturité $(20,60 \pm 2,59$ à $22,05 \pm 2,85 \mathrm{~mm})$ et la longueur $(24,19 \pm 2,70$ à $25,24 \pm 2,73 \mathrm{~mm})$ obtenues peuvent s'expliquer par la sousespèce (S. guineense subsp. macrocarpum) étudiée. De même, les diamètres et longueurs mesurés ont atteint $2 \mathrm{~cm}$ voire nettement supérieurs (Akoègninou et al., 2006 ; Arbonnier 2008 ; Eyog Matig et al., 2006). La présente étude montre que $S$. guineense subsp. macrocarpum a une fructification étalée entre 17 février et 30 avril avec la pleine fructification se situant entre mars et avril. Nos résultats corroborent bien ceux de Vayssières et al. (2010) qui ont montré que $S$. guineense se retrouve parmi une vingtaine d'espèces fruitières locales présentant des périodes de fructification qui s'échelonnent de mars à octobre en zone soudano-guinéenne du Bénin.

La faible productivité obtenue chez $S$. guineense dans les FCN et FCT peut s'expliquer par le faible taux de succès de la fructification des arbres et la dominance des rejets $(\mathrm{dbh}<10 \mathrm{~cm}$, hauteur $<1 \mathrm{~m})$, soit 1 à 13 rejets par souche avec une moyenne de $6,20 \pm 3,07$ rejets/ souche. Par contre, la production des arbres du site de Bassila (4657 fruits/ha) peut s'expliquer par la prépondérance des individus à gros diamètres ayant fleuri abondamment. Le nombre moyen de fleurs par inflorescence $(45,12 \pm 16,37)$ dans la forêt classée de Bassila est très proche de celle trouvée par Yédomonhan (2009) à Manigri-Bassila qui était de 45 fleurs/inflorescence pour une production florale correspondante par arbre qui est de 57746 fleurs/arbre (Yédomonhan, 2009). Les décalages de production florale observés dans les autres forêts sont liés aux diamètres (dbh), 
et aux nombreuses pertes d'inflorescence et d'infrutescence observés entre les stades fleurs sénescentes $\left(\mathrm{fl}_{4}\right)$ et fruits initiés $\left(\mathrm{fr}_{1}\right)$.

En effet, nos résultats ont montré d'importants avortements, pertes et dessèchements à différentes phases phénologiques. Par exemple, du stade d'initiation florale à la nouaison, 5,69\% de succès par arbre et de la nouaison aux fruits mûrs, 4,62\% de réussite. Le taux de fruits mûrs par rapport aux fleurs épanouies est de 2,55\% par arbre. Ces pertes de fleurs et de fruits au cours de la période de reproduction, ont été très importantes, et ont certes déjà été constatées chez bon nombre espèces telles que Piliostigma reticulatum, Tamarindus indica (Leguminosae - Caesalpinioideae) (Diallo et al, 2008 ; Dao, 2012).

Selon Muhanguzi et al. (2003) et Dao (2012), certaines causes comme la pollinisation insuffisante, la limitation de ressources nutritives, les attaques parasitaires, les prédations des organes de reproduction, les conditions climatiques limitantes pourraient expliquer les irrégularités de fructification et les pertes en production fruitière. D'après Orwa et al. (2009), les inflorescences de Syzygium guineense sont fréquemment attaquées par des insectes et empêchent le développement de ses fleurs. Sur cette plante vivent des Hémiptères tels que Ctenarytaina sp. Ces insectes sont responsables de la chute des bourgeons axillaires et le ralentissement de la croissance végétale (Soufo et Tamasse in Oludayo et al. 2015).

\section{Conclusion}

La présente étude a permis de mettre en évidence les phénophases florales et les potentialités fruitières de Syzygium guineense, une espèce forestière qui pourraient être exploitées pour sa domestication ou sa reproduction. De même, l'irrégularité de la fructification par endroit pourrait constituer un critère de sélection pour la production des individus par les programmes d'amélioration génétique. A priori, la floraison des individus à gros diamètres de Syzygium guineense est abondante (56460 à 193279 fleurs initiées/ arbre) dans la forêt classée de Bassila (FCB) mais la production moyenne en fruits est faible (1244 fruits/ha). Par ailleurs, le décalage enregistré entre la production de fruits et la faible densité de régénération obtenue suscite des interrogations sur l'évaluation des facteurs environnementaux de survie des graines et des plantules.

\section{CONFLIT D'INTERETS}

Les auteurs de cet article déclarent qu'il n'y a aucun conflit d'intérêts.

\section{CONTRIBUTIONS DES AUTEURS}

RBB a assuré la conception de la méthodologie, la collecte et le traitement des données puis la rédaction du manuscrit. Les autres co-auteurs ont contribué à la relecture de la méthodologie et des différentes versions du manuscrit pour l'amélioration de sa qualité scientifique.

\section{REFERENCES}

Abbad A, Benchaabane A. 2004. The phenological study of Atriplex halimus L. Notes and recors. African Journal of Ecology, 42(1): 69-73. http: //dx.doi.org/10.1111/j.01416707.2004.00449.x

Adomou AC. 2005. Vegetation patterns and environmental gradient in Benin: Implications for biogeography and conservation. $\mathrm{PhD}$ thesis, Wageningen University, the Netherlands, p. 136.

Akoègninou $\mathrm{A}$, van der Burg $\mathrm{WJ}$, van der Maesen LJG. 2006. Flore Analytique $d u$ Bénin. Backhuys Publishers: Wageningen ; 1034.

Arbonnier PBM. 2008. Ligneux du Sahel. Outil graphique d'identification V.1.0. CIRAD. 
Badou BR, Yedomonhan H. 2017. Caractéristiques structurales des peuplements ligneux à Syzygium guineense (Willd.) DC. subsp. macrocarpum (Myrtaceae) en vue de leur aménagement à des fins apicoles au Bénin. European Scientific Journal, 13(24) :186-203. URL:http://dx.doi.org/ 10.19044/esj.2017.v13n24p186.

Boffa JM. 2000. West African Agroforestry Parklands: Keys to Conservation and Sustainable Management. Unasylva English Ed. 51: 11-17.

Boshier DH. 2000. Mating systems. In Forest Conservation Genetics: Principles and Practice, Young A, Boshier D, Boyle T (eds). CSRO Publishing, CABI Publishing; 63- 80.

Chuine I, Belmonte J, Mignot A. 2000. A modelling analysis of the genetic variation of phenology between tree populations. Journal of Ecology, 88(4) : 561-570. http: //dx.doi.org/10.1046/ j.1365-2745.2000.00468.x

Dao ECM. 2012. Biologie et écologie de la reproduction sexuée d'une Caesalpinioideae (Leguminoseae) : Piliostigma reticulatum (D.C.) Hochst. Thèse de Doctorat de l'Université Polytechnique de BOBO-DIOULASSO (UPB) / Burkina - Faso. p.110.

Diallo BO, McKey D, Chevallier M-H, Joly HI, Hossaert-McKey M. 2008. Breeding system and pollination biology of the semi domesticated fruit tree, Tamarindus indica L. (Leguminosae: Caesalpinioideae): Implications for fruit production, selective breeding and conservation of genetic resources. African Journal of Biotechnology, 7(22): 4068-4075.

Diouf M, Zaäfouri MS. 2003. Phénologie comparée d'Acacia raddiana au nord et au sud du Sahara. In Un Arbre au
Désert : Acacia raddiana, Grouzis M, Le Floc'h E (éds). Ird éditions: Paris, France ; 103-118.

Djonwangwe D, Tchuenguem Fohouo F-N, Messi J, Brückner D. 2011. Foraging and pollination activities of Apis mellifera adansonii Latreille (Apidae) on Syzygium guineense var. guineense (Myrtaceae) flowers at Ngaoundéré (Cameroon) Journal of Animal \& Plant Sciences, 10(3): 1325-1333.

Ewédjè E-E, Ahanchédé A, Hardy OJ, Ley AC. 2015. Reproductive biology of Pentadesma butyracea (Clusiaceae), source of a valuable non timber forest product in Benin. Plant Ecology and Evolution, 148(2): 213-228.

Eyog Matig O, Ndoye O, Kengue J, Awono A. 2006. Les Fruitiers Forestiers Comestibles du Cameroun. IPGRI Regional Office for West and Central Africa c/o ITTA 08 B.P. 0932 Cotonou, Benin (C) International Plant Genetic Resources Institute, p. 220.

Gbesso F. 2014. Investigations ethnobotaniques, variabilités écologique et morphologique et conservation de Borassus aethiopum Mart. (Arecaceae) dans la zone soudano-guinéenne (Bénin). Thèse de Doctorat de l'Université d'Abomey-Calavi (Bénin), FAST/UAC, p. 274.

Jaouadi W, Hamrouni L, Khouja LM. 2012. Phénologie d'Acacia tortilis subsp. raddiana dans le parc national de Bou Hedma en Tunisie, effet du site sur les phénophases de l'espèce. Bois et Foêts des Tropiques, 312(2).

Kouyaté AM, Van Damme P, Diawara H. 2006. Evaluation de la production en fruits de Detarium microcarpum Guill. \& Perr. au Mali. Fruits, 61: 267-272. 
Kouyaté AM. 2005. Aspects ethnobotaniques et étude de la variabilité morphologique, biochimique et phénologique de Detarium microcarpum guill. \& perr. au Mali. Thèse de Doctorat de l'Université de Gent, 190.

Muhanguzi HDR, Obua J, Oryem-Origa H, Vetaas OR. 2003. Tree fruiting phenology in Kalinzu forest, Uganda, African Journal of Ecology, 41: 171178. http: //dx.doi.org/10.1046/j.13652028.2003.00431.x

Orwa C, Mutua A, Kindt R, Jamnadass R, Anthony S. 2009. Agroforestree Database : a tree Reference and Selection Guide version 4.0 Syzygium guineense Myrtaceae (Willd.). Agroforestry Database 4.0. DC.; 5.

Sinsin B, Kampmann D. 2010. Atlas de la Biodiversité de l'Afrique de l'Ouest (Tome I). Frankfurt/Main: Cotonou, Bénin.

Soufo L, Tamesse JL. Biologie de Blastopsylla occidentalis (Psylloidae, Aphalaridae, Spondyliaspidinae), Psylle de Eucalyptus spp. (Myrtaceae) et de Ctenarytaina sp. (Spondyliaspidinae), Psylle de Syzygium guineense (Myrtaceae) à Yaoundé (Cameroun). 197 p. In: Oludayo OE, Ngalo Otieno-Ayayo Z, Omondi Aman B, Ouali-N'goran M, Okwae Ken F, Elhassan Salah FE. 2015. Livre des Résumés 21ème Réunion et Conférence de l'Association Africaine des Entomologistes. Palais des Congrès de Cotonou, Bénin 254 p.
UICN. 2000. Red list of Threatened Species. Compiled by Craig Hilton-Taylor. The World Conservation Union. p. 38.

Vayssières J-F, Sinzogan A, Adandonon A, Ayegnon D, Ouagoussounon I, Modjibou S. 2010. Principaux fruitiers locaux des zones guinéo-soudaniennes du Bénin: inventaire, période de production et dégâts dus aux mouches des fruits. Fruit, Vegetable and Cereal Science and Biotechnology, 4(1): 42-46.

Yédomonhan H. 2009. Plantes mellifères et potentialités de production de miel en zone guinéenne et soudano-guinéenne au Bénin. Thèse de Doctorat de l'Université d'Abomey-Calavi (Bénin), FAST/UAC, p. 273.

Yédomonhan $\mathrm{H}$, Tossou MG, Akoègninou A, Demènou BB, Traoré D. 2009. Diversité des plantes mellifères de la zone soudano-guinéenne: cas de l'arrondissement de Manigri (CentreOuest du Bénin). Int. J. Biol. Chem. Sci., 3(2): $355-366$. 